\title{
Tracheal intubation
}

\author{
Toshio Matsumoto, ${ }^{1}$ Werther Brunow de Carvalho²
}

\begin{abstract}
Objective: To review current concepts related to the procedure of tracheal intubation in children.

Sources: Relevant articles published from 1968 to 2006 were selected from the MEDLINE, LILACS and SciELO databases, using the keywords intubation, tracheal intubation, child, rapid sequence intubation and pediatric airway.

Summary of the findings: Airway management in children is related to their physiology and anatomy, in addition to specific factors (inherent pathological conditions, such as malformations or acquired conditions) which have a decisive influence on success. Principal indications are in order to maintain the airway patent and to control ventilation. Laryngoscopy and tracheal intubation cause cardiovascular alterations and affect airway reactivity. The use of tubes with cuffs is not prohibited, as long as the correct size for the child is chosen. A difficult airway can be identified against the Mallampati scale and by direct laryngoscopy. Rapid sequence intubation is being recommended more and more often in pediatrics, since it facilitates the procedure and presents fewer complications. Tracheal intubation should be carried out in an adequate manner in special circumstances (eaten recently, neurological dysfunction, unstable spinal column, upper airway obstruction, laryngotracheal injuries, injuries to the eyeball). Extubation should be meticulously planned, since there is chance of failure and a need for reintubation.
\end{abstract}

Conclusions: Tracheal intubation of children requires knowledge, skill and experience, since, if the procedure is carried out by inexperienced pediatricians, it can result in life-threatening complications.

J Pediatr (Rio J). 2007;83(2 Suppl):S83-90: Intubation, tracheal intubation, child, rapid sequence intubation, pediatric airway.

\section{Introduction}

(...) but that life may in a manner of speaking be restored to the animal, an opening must be attempted in the trunk of the arteria aspera, into which a tube of reed or cane should be put; you will then blow into this, so that the lung may rise again and the animal take in the air (...). (Vesalius ${ }^{1}$; "arteria aspera" refers to the trachea.)

Tracheal intubation is the placement of a tube into the trachea, whether via the oral or nasal routes. Tracheostomy had been described before the era of Christ by the Greek physician Asclepiades. ${ }^{2}$ tracheal intubation was only described centuries later. The first known description was by
Andreas Vesalius in $1543,1,3$ carried out on an animal pneumothorax model. Vesalius can be considered a man who was well ahead of his time, because he had observed the interaction of the cardiorespiratory system. He described how, by rhythmical inflation of the lungs, pulmonary collapse was avoided and, the heart became strong and did not stop. He even described that these measures could save lives. However, it was only in 1896 that Trendelenburg ${ }^{3}$ performed the first successful tracheal intubation on anesthetized humans. He imagined a tube with an inflatable cuff at the distal end, which would make it possible to seal the airway when introduced via tracheostomy. The development of the

1. Médico. Coordenador, Unidade de Terapia Intensiva Pediátrica e Unidade de Terapia Intensiva Neonatal, Hospital Municipal Infantil Menino Jesus, São Paulo, SP, Brasil.

2. Professor adjunto livre-docente, Departamento de Pediatria, Universidade Federal de São Paulo - Escola Paulista de Medicina (UNIFESP-EPM), São Paulo, SP, Brasil. Chefe, Unidades de Cuidados Intensivos Pediátricas, Hospital São Paulo, Hospital Santa Catarina e Pronto-Socorro/Hospital Infantil Sabará, São Paulo, SP, Brasil.

Suggested citation: Matsumoto T, de Carvalho WB. Tracheal intubation. J Pediatr (Rio J). 2007;83(2 Suppl):S83-90.

doi 10.2223/JPED.1626 
laryngeal scopes with different types of blades made it possible to introduce the tube without a tracheostomy. Today many different models and sizes of laryngoscope blades are available for specific uses, including models with fiber optics. Trachea were intubation is a common procedure in emergency departments, intensive care and operating theatres. However, since it is invasive, it is not free from risks and complications. Airway management is the most essential skill in emergency medicine. ${ }^{4}$ For more than 25 years ${ }^{5}$ there has been growing concern with making this procedure as rapid and safe as possible.

\section{Anatomical considerations}

Knowledge of the airway anatomy is of prime importance to the success of tracheal intubation. Attempting airway access without prior anatomical knowledge can result in serious consequences. The airways of children differ from those of adults and undergo significant changes from birth to school age. Some of these peculiarities ${ }^{2}$ are described in Table 1 and are the reasons for the differences in intubation technique for different age groups.

The shape of the larynx merits special attention. For more than 50 years it has been well-known, in the majority of texts on children's airways, that the shape of the larynx is conical, with narrowing in the caudal direction. In 2003, Litman et al. ${ }^{6}$ published a challenging article. They observed the transverse and anteroposterior diameters of the larynges of 99 children (0-14 years) who underwent elective magnetic resonance imaging. The children were in deep sedation with propofol, without curarization and were breathing spontaneously. In this condition, examination of the larynx images demonstrated that, in all of the children, the narrowest point of the larynx was at the level of the vocal chords. Furthermore, the shape of the larynx in the transverse diameter was conical with the apex of the cone exactly at the vocal chords, i.e. the opposite of what is described. In the anteroposterior direction, the shape if cylindrical, with no change in diameter along the larynx. Despite these surprising data, the authors suggest that this divergence could be provoked by the action of laryngeal musculature in sedated patients without curarization and that the structures above the cricoid may have been distended since they do not have cartilage all around their circumference. Thus, functionally, the rigid cricoid ring would still be the narrowest point of the larynx.

The upper airway consists of the path taken by air from the nostrils and mouth to the trachea. It is divided into nasopharynx, oropharynx and pharynx. The nasopharynx comprises the nasal cavity, septum, nasal conchae and adenoids. The oropharynx includes the oral cavity oral cavity, teeth and tongue. The pharynx includes the tonsils, uvula and epiglottis. The epiglottis separates the larynx from the hypopharynx.

The larynx is the route to the trachea, and the hypopharynx is the route to the esophagus. The larynx is the target structure in tracheal intubation (also known as

Table 1 - Anatomical peculiarities of the upper airways of children compared with adults

\begin{tabular}{ll}
\hline Anatomical peculiarities & Clinical significance \\
\hline
\end{tabular}

Head proportionally larger, about $1 / 4$ of the body at birth

Prominent occiput

Relative macroglossia

Epiglottis is shorter and narrower and is angled with relation to the axis of the trachea

Conical larynx (up until around 10 years of age), in contrast with the cylindrical adult shape
Difficulty with achieving correct positioning for intubation

Difficulty with achieving correct positioning for intubation

Greater resistance to the passage of air. Can make placement of laryngoscope blades difficult. Posterior displacement of the tongue can aggravate the problem

It is difficult to see this point of reference

The narrowest point of the larynx is in the subglottic area. In adults, this point is in the glottic area. The higher larynx position makes the angle between the base of the tongue and the glottis more acute. It is easier to see the glottis in this situation using straight blades 
translaryngeal) and can be separated into the supraglottic and subglottic sections, separated by the glottis, where the vocal chords are located. The glottic structure is shown in Figure 1.

\section{Indications for tracheal intubation}

Emergency medicine begins with the airway. ${ }^{7}$ In any situation in which it is necessary to maintain a patent and safe airway, tracheal intubation may be indicated. Therefore, tracheal intubation is indicated for those patients who need a patent airway to be maintained and pulmonary ventilation controlled.

The procedure may be of an emergency or an elective character. Indication of the elective mode is primarily reserved for patients undergoing surgery, and this is a decision taken by anesthetists and endoscopists. For the pediatrician, principal indications are in emergency situations such as cardiorespiratory arrest, respiratory insufficiency, hypoventilation, shock, coma, postoperative care and multiple traumas. Tracheal intubation may also be used to control ventilation $\left(\mathrm{PaCO}_{2}\right)$ and to administer medications such as surfactant and those indicated for cardiorespiratory arrest. We can consider that any patient requiring ventilatory support by means of pulmonary ventilation mechanical has an indication for tracheal intubation.

Tracheal intubation should always be performed by skilled and experienced personnel.

\section{Physiological effects of tracheal intubation}

Tracheal intubation provides an artificial airway which makes pulmonary ventilation possible free from upper airway obstructions. Nevertheless, the tube may create increased airflow resistance. the resistance to airflow in a tubular system is predicted by Poiseuille's law (equation 1). According to this law, resistance is inversely proportional to the radius to the power of four. Therefore, the airway diameter is reduced by the presence of the tube wall.

\section{Equation 1 - Poiseuille's law}

$$
\begin{aligned}
& \text { Flow }=\frac{\mathrm{P} \times \Pi \times \mathrm{r}^{4}}{8 \times \mathrm{I} \times \mathrm{n}} \\
& \mathrm{P}=\text { pressure } \\
& \mathrm{r}=\text { radius } \\
& \mathrm{I}=\text { distance } \\
& \mathrm{n}=\text { viscosity }
\end{aligned}
$$

Since resistance $=$ pressure $/$ flow, therefore

Resistance $=8 \times \mathrm{I} \times \mathrm{n}$

$$
\overline{\Pi \times r^{4}}
$$

The diameter normally used for reference is the internal diameter (ID), but the outside diameter (OD) of the tube is the factor limiting its insertion into the larynx. Depending on the manufacturer, tubes with the same ID can have different ODs, i.e. different thicknesses. The longer the tube, the greater the resistance imposed, and this increases further if there are bends or narrowing due to obstructions within the tube. This means that an intubated child will perform greater respiratory work when breathing spontaneously. The endotracheal tube can be made from a variety of plastic materials, as long as they are inert, nontoxic, do not induce foreign body reactions and are non-traumatic. The physical characteristics of the tube are also important, since it must not be too rigid in order not to traumatize airways, nor must it be too malleable, or it will get bent and its caliber reduced by external compression. The choice of correct endotracheal tube size should be based on the diameter of the cricoid region (subglottic), since this is the point of the greatest functional narrowing.

Tubes with cuffs are not prohibited for pediatric use, ${ }^{8,9}$ except during the neonatal period, as long as the relationship between the $O D$ and the larynx is respected. In lung diseases with low complacency or high airway resistance and a requirement for higher inspiratory pressures or when there are major air leaks past the tube, a cuffed tube is preferable. Cuff pressure should not exceed $20 \mathrm{cmH}_{2} \mathrm{O}$.

Tracheal intubation eliminates the participation of the upper airways, and the air inhaled enters the lower respiratory tract directly without going through the normal process of conditioning. This conditioning of inhaled gas (filtration, heating and humidification) is important to the integrity of the airway. Inadequately conditioned gas can provoke injuries related to the production of mucus and mucociliary movement, in addition to favoring pulmonary infections. ${ }^{10,11}$

Exclusion of the glottis with the air passing through the translaryngeal tube removes the possibility of positive pressure during the expiratory phase. Occlusion of the glottis combined with contraction of the abdominal muscles generates positive pressure in the airways, acting as a Valsalva maneuver. Therefore, in lung diseases with a tendency to alveolar collapse (for example, respiratory distress syndrome), this mechanism, characterized by expiratory grunting to maintain alveoli open, is compromised after intubation. ${ }^{12}$ In this state, pulmonary collapse is faster, if positive expiratory pressure is not added via the endotracheal tube.

\section{Recognition of the difficult airway}

One of the situations most feared by physicians is to be faced with a child requiring ventilatory life support and be unable to achieve successful tracheal intubation after at least 


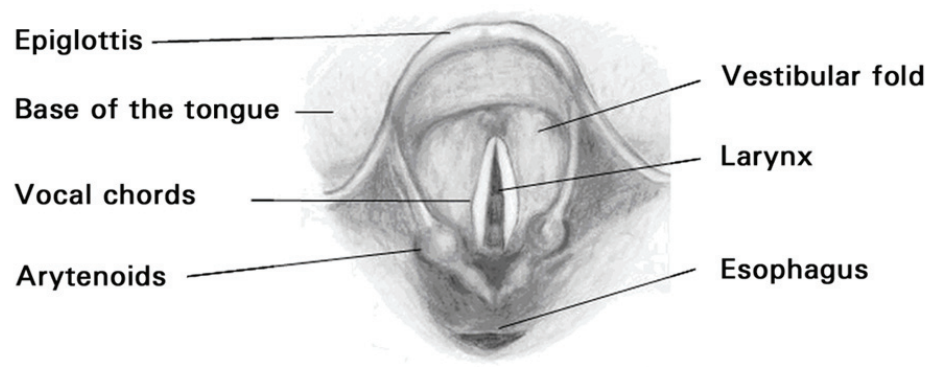

Figure 1 - View of the glottic area via direct laryngoscopy

two attempts. Some of these situations are the result of a lack of technical ability, but, in a small number of others, the cause is a difficult airway. The difficult airway does not have a standard definition. ${ }^{13}$ According to the American Society of Anesthesiologists, ${ }^{13}$ "a difficult airway is defined as the clinical situation in which a conventionally trained anesthesiologist experiences difficulty with face mask ventilation of the upper airway, difficulty with tracheal intubation, or both. The difficult airway represents a complex interaction between patient factors, the clinical setting, and the skills of the practitioner." ${ }^{13}$ Among patient factors, there are certain obvious clinical signs that predict difficult tracheal intubation, particularly malformations, tumors and facial traumas. In attempts to predict the difficulty that will be encountered during intubation, scores have been created for use with adults. Mallampati, ${ }^{14}$ in 1985 , described a graded system based on preoperative observation of the oropharynx of the patient while seated. The patient extends their tongue as far forward as possible and observations are made of the structures of the pharynx (tonsillar pillars, soft palate and base of the uvula). Depending upon what can be seen in this test, a classification is made. Figure 2 illustrates this classification, as modified by Samsoon \& Young. ${ }^{15}$

View of pharynx during the test: 1 ) soft palate, tonsillar pillars and uvula visible; 2 ) soft palate and uvula visible; 3 ) soft palate and base of the uvula visible; 4 ) soft palate is not visible.

Patients with in classes III and IV have a greater risk of presenting difficulties during tracheal intubation.

The application of this test to pediatrics should be careful, since children's anatomy alters with age and the degree of patient cooperation is unpredictable.

One meta-analysis ${ }^{16}$ demonstrated that this modified test had good correlation for predicting difficulties in laryngoscopy and intubation. Nevertheless, it offered limited accuracy for predicting a difficult airway when used in isolation.
Another scale described by Cormack \& Lehane ${ }^{17}$ in 1984 with obstetric patients, is based on viewing the glottic region with direct laryngoscopy and classifying according to the structures that are visible (glottis, arytenoids and epiglottis): I - view of entire glottis; II - partial view of glottis and arytenoids; III - only glottis visible; IV - neither glottis nor epiglottis visible. Patients at degrees III and IV present a higher risk of difficult intubation. The need for direct laryngoscopy limits prediction of the airway prior to the intubation procedure.

\section{Preparation of the patient and technique}

\section{Positioning}

Placement of a small cushion under the heads of older children in order to facilitate positioning of the axes (Figure 3).

With newborn infants, the cushion under the head is often unnecessary because of the large size of the head, proportionally, where the occiput already raises the level of the head. In these cases, a cushion may be used under the neck and shoulder blades to stabilize the head position.

\section{Intubation}

The laryngoscope blade most used in pediatrics is the straight type (Miller). After positioning, the laryngoscope is held in the left hand and the blade introduced laterally at the right side of the mouth, attempting to displace the tongue to the left. The blade is smoothly and lightly pushed along the tongue until the epiglottis can be seen. The blade should overlap the epiglottis in order to view the glottic region (Figure 3C). When using a curved blade, the distal extremity is placed between the base of the tongue and the vallecula.

\section{Rapid sequence intubation}

The term appeared in anesthesia during the 1980s as an extension of the rapid sequence anesthetic induction. It was observed that the use of pre-medication facilitated the process and made it safer and with a higher rate of success. In 1990, Yamamoto ${ }^{18}$ reported successfully using rapid 


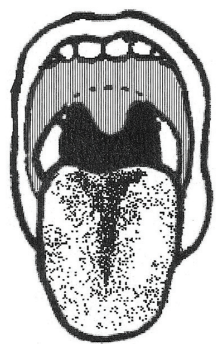

1

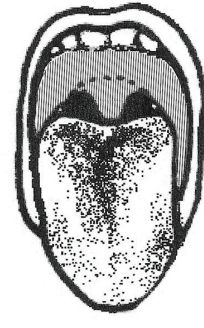

2

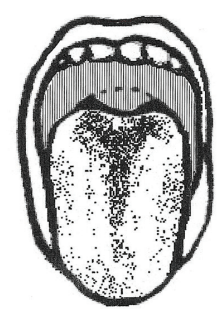

3

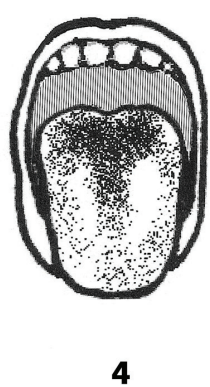

4

Figure 2 - Mallampati classification, modified by Samsoon \& Young ${ }^{15}$

sequence intubation (RSI) with 19 pediatric patients requiring emergency tracheal intubation.

The details of RSI do not fall within the scope of this article, and can be found by consulting articles specifically on that subject. ${ }^{8,13,19-21}$ Briefly, it consists of the administration, after a period of oxygenation at $100 \%$, of a rapid acting and short duration sedative, followed by a neuromuscular blocking agent with the same characteristics, in addition to applying pressure to the cricoid cartilage (Sellick maneuver), in order to carry out laryngoscopy rapidly and under the best conditions, followed by oral endotracheal intubation. The aim is to avoid unsuccessful attempts and manual ventilation with bag and mask, reducing the risk of distension, regurgitation, vomiting and aspiration. ${ }^{21}$

\section{Special circumstances for relevant to tracheal intubation}

Children who have eaten a short time previously are at great risk of aspiration of gastric content during handling and tracheal intubation, particularly in cases where airway protection reflexes are abnormal. The risk factors related to morbidity are: aspirate volume, $\mathrm{pH}$ of aspirate $\mathrm{pH}$ lower than 1.8 is associated with increased pulmonary dysfunction and death), ${ }^{22}$ bacterial contamination, food particles and food types, prior systemic or pulmonary disease and immunodepression. Rapid sequence intubation is formally indicated for these patients.

In patients with severe central nervous system dysfunction (trauma, hemorrhage, infection, hydrocephalus or mass lesions), there is a risk of the combination of intracranial hypertension and cerebral herniation, with tracheal intubation and the institution of mechanical ventilation indicated for patients with a Glasgow coma score of less than $9 .{ }^{23}$ Nasotracheal intubation is contraindicated for patients with fractures at the base of the skull and who present cerebrospinal fluid leakage, due to the increased risk of infection and even of perforation of the cribiform plate. In children with intracranial hypertension, soon after tracheal intubation and starting ventilation, the aim should be to maintain partial $\mathrm{CO}_{2}$ pressure at values of approximately 30 to $35 \mathrm{mmHg}{ }^{24}$

In patients with spinal column, the maneuvers of neck flexion and head extension could aggravate an existing injury and precipitate the possibility of further problems. Spinal column X-rays and the history of the trauma should aid to define the type of injury and the maneuvers necessary, but this information is rarely complete, meaning that the handling involved in intubation is controversial in these situations. ${ }^{25,26}$ Blind nasotracheal intubation is not recommended with these patients, and the head and neck should always be maintained immobilized in the neutral position, with the aid of an assistant.

Children intubated for upper airway obstruction due to edema, inflammation, trauma or other injuries that occupy space, should, wherever possible, be intubated via the oral endotracheal route, in a supine position or at $30^{\circ}$, performing the maneuver without neuromuscular blocking agents. Notwithstanding, the ideal conditions would be intubation in an operating theatre, using an anesthetic, with the patient breathing spontaneously, receiving a high concentration of oxygen and in deep anesthesia. In the majority of cases, the endotracheal tube should be 0.5 to $1 \mathrm{~mm}$ smaller than that predicted for the age, and there must be no air leakage. If air is leaking past the tube, extubation is indicated.

In children with open injuries to the eyeball who require intubation for associated injuries, the procedure should be performed with the objective of preventing increase in ocular pressure, which could cause extrusion of the vitreous matter and blindness. Intubation should be careful and smooth, using muscle relaxant at taking care not to apply any pressure to the affected eye with the mask. Succinylcholine should not be used, since it is associated with increased intraocular pressure. In general, agents that depress the central nervous system reduce intraocular pressure, with the exception of ketamine. Lidocaine supplements the effect of 


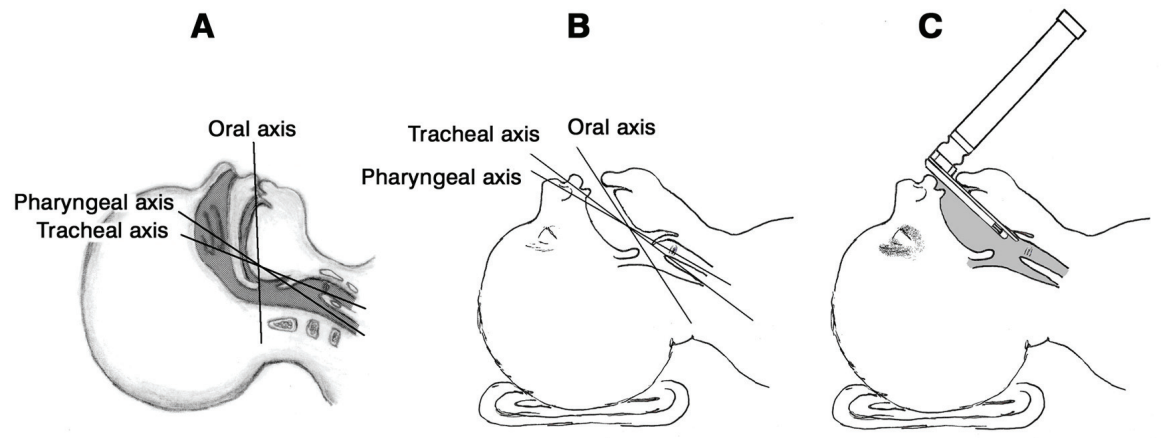

Figure 3 - A) Illustration of the axes (oral, pharyngeal and tracheal); B) alignment of these axes with correct positioning; $C$ ) viewing the glottic fold with a straight blade

sedative and analgesic agents, blocking increase in intraocular pressure. Deep sedation and paralysis should be maintained until surgical correction of the eye injury has been performed.

In children with laryngotracheal injuries with a history of trauma to the anterior part of the neck, and clinical findings of hoarseness, stridor, subcutaneous emphysema, pneumothorax or pneumomediastinum, tracheal intubation should be performed with the patient awake, but with careful sedation and topical anesthesia. Observation is direct, via laryngoscopy or fibrobronchoscopy, aiming to avoid the risk of complete and sudden obstruction of the airway, or the creation of a false path through the airway.

\section{Handling tracheal extubation}

Extubation is the final phase in the process, with successful or otherwise protection of the airway and/or treatment with mechanical ventilation. However, the success of extubation depends on several different factors, in addition to the resumption of pulmonary function. Clinicians need the ability to accurately assess the readiness of a child for extubation, to manage the whole extubation process correctly and to identify and immediately deal with potentially grave post-extubation complications.

The employment of corticosteroids is controversial, since the different studies on the subject did not differentiate between different causes of upper airway obstruction. 27,28 We have been using dexamethasone $(0.25 \mathrm{mg} / \mathrm{kg}$ /intravenous dose) with patients where the endotracheal tube air leak test suggests a high risk of upper airway obstruction after extubation, and also in children with risk factors (traumatic intubation, multiple intubations, prolonged intubation, surgical procedures in the cervical or laryngeal regions). Corticoid is prescribed approximately 24 hours before extubation and maintained until 24 hours after extubation. Children less than four years old are more likely to develop edema of the airway, due to contributing factors, such as a narrow or cuffed tracheal tube, excessive movement of the tube or the patient due to inadequate sedation or analgesia, traumatic intubation or multiple intubation attempts, prior airway abnormalities and airway infections. ${ }^{29}$ The great majority are mild cases and treated with warmth and humidity. For more severe cases, we routinely use inhaled epinephrine at a dosage of $0.25 \mathrm{~mL} / \mathrm{kg} /$ dose, since it is safe and effective and costs less than racemic epinephrine. Attention should be given to the possibility of relapse, and the patient continuously monitored.

In the most serious cases of upper obstruction, a helium-oxygen mixture can be used (20-30\% oxygen and $70-80 \%$ helium), aiming at the clinical improvement of the patient (reducing airway resistance and respiratory work). Helium has a lower density than air, and mixing it with oxygen allows for increased inspiratory flow and reduced resistance.

A report by Otherson, ${ }^{30}$ in 1979, suggested that restricting fluids could promote tissue dehydration and facilitate reduction of subglottic edema. Although this strategy is useful for high risk patients, it is not our routine.

The presence of an endotracheal tube can temporarily alter the reflexes of the larynx and the function of vocal chords. The tendency to deglutition of air during periods of respiratory distress can also contribute to an increased risk of vomiting and aspiration. We have maintained patients in fast for 4 to 6 hours prior to extubation and, when necessary, we have emptied stomach contents using a nasogastric tube. The preparations for extubation also include preparations for possible reintubation, with the medications and materials for that procedure being made available. A small percentage of patients will require reintubation after extubation, and the incidence is greater in cases where intubation was difficult. ${ }^{31}$ 
Patients should only be reintubated if pharmacological treatment was ineffective. In cases where there is a risk of reintubation it is recommended that extubation be performed with a fiber optic bronchoscope available. The procedure for extubation includes: a) placing the child in a semi-erect position; b) careful aspiration of the endotracheal tube, and also the upper airway (mouth and nose); c) oxygenation of the patient with $\mathrm{O}_{2}$ at $100 \%$, for 3 to 5 minutes, using a bag-mask with reservoir; d) attention: deflate the cuff of the endotracheal tube; and) request preschool children and older to cough as the tube is removed or remove the tube during the inspiratory phase; f) encourage deep breathing coughing immediately after removal of the tube.

\section{Complications}

The most common complications after tracheal extubation are compromised upper airways and atelectasis. After extubation, continuous monitoring and early recognition and treatment of potential complications are obligatory. Respiratory and heart rates should be monitored, together with pulse oximetry and expired carbon dioxide providing important data on worsening ventilatory function which could be related to post-extubation complications.

Patients who develop severe subglottic edema manifests symptoms during the first 2 hours after extubation, and obstruction generally tinges its maximum of at 8 to 12 hours. Children who do not have symptoms during the first 2 hours will generally not develop a severe obstruction. For patients at greater risk, employ inhaled racemic adrenaline or adrenaline 1/1,000, even before onset of symptoms. Treatment of post-extubation subglottic edema is with corticoid and adrenaline. A helium-oxygen mixture (70\% helium: $30 \%$ oxygen) can be used with patients with moderate-severe edema subglottic. This strategy has helped to prevent additional trauma due to reintubation of those patients where treatment for upper airway obstruction fails. ${ }^{32,33}$ When reintubation is inevitable, an endotracheal tube should be chosen $0.5 \mathrm{~mm}$ smaller (or smaller still) than that previously employed. A second attempt should be planned 48 hours after instituting corticoid. Consider performing a bronchoscopy, which may reveal the presence of tracheal stenosis, granulomas, ruptured arytenoid cartilage, ulceration/altered mobility of the vocal chords. Paralysis of the vocal chords is associated with increased risk of pulmonary aspiration, and recognition of this paresis is important to reduce this possibility.

One relatively common complication is post-extubation atelectasis related to altered mucociliary clearance during and immediately after extubation and associated with unresolved preexisting lung disease. It most often affects the upper lobe of the right lung. The intensive care specialist should anticipate the possibility of collapse by means of clinical assessment or chest X-rays of the patient after extubation. Respiratory physiotherapy should always be assessed individually for each patient, depending on clinical conditions, and can be indicated before extubation or afterwards to improve the ventilatory conditions and pulmonary mechanics of the child.

\section{References}

1. Vallejo-Manzur F, Perkins $Y$, Varon J, Baskett P. The resuscitation greats: Andreas Vesalius, the concept of an artificial airway. Resuscitation. 2003;56:3-7.

2. Cordeiro AMG. Acessos para as vias aéreas. In: Carvalho WB, Hirschheimer MR, Matsumoto $T$, editores. Terapia intensiva pediátrica. 3a ed. São Paulo: Atheneu; 2006. p. 1589-605.

3. Dunn PF, Goulet RL. Endotracheal tubes and airway appliances. Intern Anesth Clin. 2000;38:65-94.

4. Walls RM. Rapid-sequence intubation comes of age. Ann Emerg Med. 1996;28:79-81.

5. Graham CA. Advanced airway management in the emergency department: what are the training and skills maintenance needs for UK emergency physicians? Emerg Med J. 2004;21:14-9.

6. Litman RS, Weissend EE, Shibata D, Westesson PL. Developmental Changes of laryngeal dimensions in unparalyzed, sedated children. Anesthesiology. 2003;98:41-5.

7. Reid C, Chan L, Tweeddale M. The who, where, and what of rapid sequence intubation: prospective observational study of emergency RSI outside the operating theatre. Emerg Med J. 2004;21:296-301.

8. International Liaison Committee on Resuscitation (ILCOR). Guidelines for Pediatric Cardiopulmonary Resuscitation and Emergency Cardiovascular Care. Circulation. 2005;112 Suppl $1: 167-87$.

9. Weiss M, Gerber AC. Cuffed tracheal tubes in children - things have changed. Pediatr Anesth. 2006;16:1005-7.

10. Shelly MP, Nigthngale P. ABC of intensive care: respiratory care. BMJ. 1999;318:1674-7.

11. Nakagawa NK, Franchini ML, Driusso $P$, de Oliveira LR, Saldiva $\mathrm{PH}$, Lorenzi-Filho G. Mucociliary clearance is impaired in acutely ill patients. Chest. 2005;128:2772-7.

12. Harrison VC, Heese $\mathrm{H}$ de $\mathrm{V}$, Klein $\mathrm{M}$. The significance of grunting in hyaline membrane disease. Pediatrics. 1968;41:549-59.

13. American Society of Anesthesiologists Task Force on Difficult Airway Management. Practice guidelines for management of the difficult airway: an updated report by the American Society of Anesthesiologists Task Force on Management of the Difficult Airway. Anesthesiology. 2003;98:1269-77.

14. Mallampati SR, Gatt SP, Gugino LD, Desai SP, Waraksa B, Freiberger $D$, et al. A clinical sign to predict difficult tracheal intubation: a prospective study. Can Anaesth Soc J. $1985 ; 32: 429-34$.

15. Samsoon GL, Young JR. Difficult tracheal intubation: a retrospective study. Anaesthesia. 1987;42:487-90.

16. Lee A, Fan LTY, Gin T, Karmakar MK, Ngan Kee WD. A systematic review (meta-analysis) of the accuracy of the Mallampati Tests to predict the difficult airway. Anesth Analg. 2006;102:1867-78. 
17. Cormack RS, Lehane J. Difficult intubation in obstetrics. Anaesthesia. 1984;39:1105-11.

18. Yamamoto LG, Yim GK, Britten AG. Rapid sequence anesthesia induction for emergency intubation. Pediatr Emerg Care. $1990 ; 6: 200-13$.

19. Amantéa SL, Piva JP, Zanella MI, Bruno F, Garcia PC. Acesso rápido à via aérea. J Pediatr (Rio J). 2003;79 Supl. 2:S127-38.

20. Reynolds SF, Heffner J. Airway management of the critically ill patient. Chest. 2005; 127:1397-412.

21. Jaguim M. Airway management. Rapid-Sequence intubation in trauma patients. AJN. 2003;103;32-5.

22. James CF, Modell JH, Gibbs CP, Kuck EJ, Ruiz BC. Pulmonary aspiration: effects of volume and $\mathrm{pH}$ in the rat. Anesth Analg. $1984 ; 63: 665-8$.

23. Guidelines for the acute medical management of severe traumatic brain injury in infants, children, and adolescents. Chapter 17. Critical pathway for the treatment of established intracranial hypertension in pediatric traumatic brain injury. Pediatr Crit Care Med. 2003;4(3 Suppl):S65-7.

24. Adelson PD, Bratton SL, Carney NA, Chesnut RM, du Coudray $\mathrm{HE}$, Goldstein B, et al. Guidelines for the acute medical management of severe traumatic brain injury in infants, children, and adolescents. Chapter 12 . Use of hyperventilation in the management of severe pediatric traumatic brain injury. Pediatr Crit Care Med. 2003;4(3 Suppl):S45-8.

25. Knopp RK. The safety of orotracheal intubation in patients with suspected cervical-spine injury. Ann Emerg Med. 1990;19:603.

26. Rhee KJ, Green W, Holcroft JW, Mangili JA. Oral intubation in the multiply injured patient: the risk of exacerbating spinal cord damage. Ann Emerg Med. 1990;9:511-4.
27. Anene $\mathrm{O}$, Meert $\mathrm{KL}$, Uy $H$, Simpson $P$, Sarnaik AP. Dexamethasone for the prevention of postextubation airway obstruction: a prospective, randomized, double-blind, placebo-controlled trial. Crit Care Med. 1996;24:1666-9.

28. Tellez DW, Galvis AG, Storgion SA, Amer HN, Hoseyni M, Deakers TW. Dexamethasone in the prevention of post-extubation stridor in children. J Pediatr. 1991;118:289-94.

29. Koka BV, Jean IS, Andre JM, MacKay I, Smith RM. Post-intubation croup in children. Anesth Analg. 1977;56:501-5.

30. Otherson HB. Intubation injuries of the trachea in children: management and prevention. Ann Surg. 1979;189:601-6.

31. Hammer GB, Funck N, Rosenthal DN, Feinstein JA. A technique for maintenance of airway access in infants with a difficult airwayfollowing tracheal extubation. Paediatr Anaesth. $2001 ; 11: 622-5$.

32. Kemper KJ, Izenberg S, Marvin JA, Heimbach DM. Treatment of post-extubation stridor in a pediatric patient with burns: the role of heliox. J Burn Car Rehabil. 1990;11:337-9.

33. Kemper KJ, Ritz RH, Benson MS, Bishop MS. Helium-oxygen mixture in the treatment of post-extubation stridor in pediatric trauma patients. Crit Care Med. 1991;19:356-9.

Correspondence:

Werther Brunow de Carvalho

Rua Correia de Lemos, 153/71, Chácara Inglesa

CEP 04140-000 - São Paulo, SP - Brazil

Tel.: +55 (11) 2276.1790

Fax: +55 (11) 5576.4288

E-mail: wertherbru.dped@epm.br 